\title{
Randglossen von Dantes Hand? ${ }^{1}$
}

Obwohl der provenzalischen Handschrift Vat. $3207(\mathrm{H})$ bereits von Dr. Grützmacher eine ausführliche Beschreibung zu Teil geworden ist ${ }^{2}$, zu der Bartsch ${ }^{3}$ einen kurzen Nachtrag geliefert hat, bleibt doch noch Einiges von ihr zu berichten. Zunächst ist es für mich eine grofse Freude, dafs ich im Stande bin, ein Resuliat der scharfsinnigen Untersuchungen Mussafias über die von Giov. Maria Barbieri benutzten provenzalischen Handschriften ${ }^{3}$ durch Angabe einiger äufserer Umstände zweifellos zu machen. Mussafia hatte gezeigt, dals das von Barbieri häufig citierte libro slegato nur $\mathrm{H}$ oder eine Abschrift von $\mathrm{H}$ sein könnte. Der Katalog des F. Ursinus ${ }^{5}$ belehrt uns nun, dafs das Erstere der Fall ist, indem er die Angabe enthält, dafs $\mathrm{H}$ ungebunden (senza coperta) in die Vaticana gelangt ist, was aufserdem noch dadurch, da/s in der Zwischenzeit viele Blätter verloren gegangen sind und dafs - der gegenwärtige Einband der der Vaticana ist, bestätigt wird. Mussafia und nach ihm Gröber 6 mufsten nach der ihnen zu Gebote stehenden Beschreibung mit Recht Anstand nehmen, $\mathrm{H}$ mit dem libro slegato zu identifizieren, weil die von Barbieri citierten Seitenzahlen teilweise mit denen vọn $\mathrm{H}$ nicht übereinstimmten. Bei genauerer Prüfung aber ergiebt sich, dafs die gegenwärtige Paginierung der Hs. eine moderne ist und dafs Barbieri die letztere noch in ziemlich vollständiger Gestalt gekannt, während sie heute mindestens ein Viertel ihres ursprünglichen Umfanges eingebürst hat. Dazu

' Die Fortsetzung des im vorigen Hefte begonnenen Artikels werde ich nicht folgen lassen, nachdem ich erfahren habe, dafs Herr de Nolhac in seinem Buche über F. Ursinus auch die romanischen Hss. zu behandeln gegedenkt. Dagegen hat derselbe mir das Studium der Petrarca- und der provenzalischen Codices ausschliefslich überlassen. Siehe auch Deutsche Litteratur-Zeitung 1886 No. 38 und 39.

2 Archiv für d. Stud. d. n. Sprachen 1863, S. $385 \mathrm{ff}$.

3 Jahrbuch f. rom. u. engl. Litt. XI 22.

- Sitzungsberichte f. histor. Kl. der Wiener Akademie der Wissenschaft LXXVI $201 \mathrm{ff}$.

5 S. diese Zeitschrift X 207.

- Die Liedersammlungen der Troubadours in Böhmers Roman. Studien II 407.

Ma le sue cansoni sono cosi difficili da intendere, che alcune si trovano essere state anticamente chiosate di comento latino per maggiore intelligenza ... Barbieri, dell' origine della poesia rimata p. 97. 
kommt, dafs Barbieri von einem lateinischen Kommentar der Gedichte Arnaut Daniels spricht, welchen er in seinen Hss. gefunden habe 1 und dafs $\mathrm{H}$ nicht nur einen solchen enthält, sondern dafs die wenigen von Barbieri citierten Stellen wörtlich mit den Glossen dieser Hs. übereinstimmen.

Diese Randglossen sind bisher wenig beachtet worden. Warum Grützmacher sie nicht abgeschrieben hat, läfst er durch seine Worte: „noch schwerer sind die erklärenden Bemerkungen am Rande der ersten Bogen $\mathrm{zu}$ entziffern" 2 deutlich erkennen. In der That ist die Schrift an sich sehr fein und durch die Zeit stark verblafst, jedoch ist es mir, indem ich mir Vormittage aussuchte, an welchen das Licht besonders günstig war, gelungen, ziemlich Alles zu lesen. ${ }^{3}$

Die Bemerkungen sind zweierlei Art: dic einen sind, meistens durch das Wörtchen uel eingeführte Textvarianten von der Hand des oder vielmehr der Schreiber; die andern sind Wort- und Sacherklärungen und Angaben von Parallelstellen, zumeist in lateinischer Sprache, teils in Minuskel, teils in Kursiv geschrieben, die aber beide von derselben Hand herrühren, da sie innerhalb derselben Bemerkung abwechseln. Ich berücksichtige nur die letzteren und gebe sie, da die Auflösung der Abkürzungen keine Schwierigkeit bot, ohne dieselben und in gewöhnlicher Schrift. Überall, wo die Lesung mir zweifelhaft war, habe ich dies durch Beisetzung von Fragezeichen angedeutet. Auf welche Stellen sich die einzelnen Erklärungen beziehen, ist in der Hs. häufig durch Häkchen bezeichnet; wo diese fehlten, wird es mir, hoffe ich, trotzdem gelungen sein, die richtigen Beziehungswörter zu finden. Was in Klammern steht, sind die von mir hinzugefügten Textstellen; dann folgen die Randbemerkungen möglichst getreu, jedoch hat mich die schlechte Konservierung derselben manchmal $\mathrm{zu}$ indovinatorischer Ergänzung genötigt.

\section{A) zu Arnaut Daniels Gedichten.4 \\ c 9 B zum Gedicht Canso dol mot (Can. II). \\ 1. V. I7 (la sec atrail) sic dat(?) atraza. ut canis sequitur lo traill del porc : la traza.}

1 Ich begnüge mich hier mit dieser allgemeinen Angabe, weil ich an anderem Orte noch speziell über die von Barbieri benutzten Hs. zu sprechen haben werde.

2. 1. c. S. 385 .

${ }^{3} \mathrm{Zu}$ meinen Bedauern ist es mir jedoch unmöglich gewesen, meine $\mathrm{Ab}$ schrift der Glossen vor dem Druck noch einmal mit dem Ms. zu vergleichen; ich mufs mir daher etwaige Ergänzungen und Berichtigungen für später vorbehalten. Ebenso ist es mir versagt gewesen, eine Reihe von Büchern zu konsultieren, da diejenige italienische Bibliothek, die mir zur Verfügung stand, überaus lückenhaft ist.

$4 \mathrm{H}$ ist in zwei Kolonnen geschrieben; $A$ und $B$ bezeichnen die des recto, $C$ und $D$ die des verso der Folii. Der leichteren Auffindmng halber habe ich bei den Gedichten Arnaut Daniels die Verszahl nach der Ausgabe Canellos hinzugefügt, und nur dann weggelassen, wenn die Bezeichung nicht klar war. 
2. W.25 (lagrim) lacrimetur.

3. W.26 (Et arderim) in ferxurio se comburat.

4. V.35 (noirim) : nuiritura.

5. V.37 (Si bem uau per tot aesdaill) sicut bestia defligala . car tu non sai on mi uai.

6. W.40 (quenz fim) fim : fecimus.

7. W.57 (laios honor) ut uos honorei.

c $9 \mathrm{C}$ zum Gedicht Sim fos Amors (Can. XVII).

8. W. 3 (Ja de mon jor nom calgra far unbarc) Aillors ditz. Ja per gran ioi nom calgra far embarc : debita so es sui ebre (?) don eu fos embariatz. so es embrigatz per debita.

9. W. 9 (no membarga) non me impedit.

Io. V. ig (sofrel parc) parco go es perdono.

I 1. V. 20 (encomba) en ualle.

12. V. 21 (pres un tom) En respeit de lei.

I3. V. j6 (retomba) augasta (?).

14. V. 37 (del som) del cap. que es la partz que es el som.

I5. V. 45 (E per vos es casutz pretz e iouens) Aillors ditz. Malsastres es qeus te desconoissens. Qe etc.

c $9 \mathrm{D}$ zum Gedicht Laura amara (Can. IX).

16. V. 18 (Tan fo clara ma prima lutz) : subtile.

17. V. 25 (daltra seslutz) illuminat.

I 8. V. 39 (Tals detz pecs) talia decem ut ita dixerim peccata.

19. V. 4 I (trencs): incidas de tronco cass[um].

20. V. 5 I (goma) co es specia.

21. V. 52 (Sim anpara) sim mante.

22. V. 56 (quetz) qetz : qietos.

23. V. $5^{8}$ (arencs) di arengar: in ordinem ponere.

24. V. 73 (etz) etz : estis.

25. V. 74 (decs) decs : finem(?) ultimum quem non licet uenire(P).

26. V. 75 fadencs so es stultitias.

27. V. 83 ufaut ue da ufana. cosa d'uana gloria.

28. V. 83 (cel de doma) Doma es us mons fort autz on es solamen una maissos de... spiritals fort (?).

c IoB zum Gedicht En cest sonet coind eleri (Can. X).

29. V. 3 (aleri) : alegro. ${ }^{1}$

30. V. 22 (queri) : quero.

31. V. 23 (toli) : tollo.

1 Dies eine Wort ist in einer der des Schreibers des Textes sehr ähnlichen Minuskel geschrieben. $\mathrm{Da}$ es dessen Gewohnheit jedoch nicht ist, derartige erklärende Bemerkungen zu machen, so müssen wir auch dieses Wort dem Glossator zuschreiben, der kurz darauf andere italienische Wörter gebraucht. Er hat wahrscheinlich anfangs in seinen Glossen die Schrift des Textes nachahmen wollen, diese Absicht aber bald wieder aufgegeben.

Zeitschr. f. rom. Phil. X. 
32. V. 26 Sesaura : saurat. sicut sparauerius cobrador hoc ued hoc operat...

33. V. 32 (lo cors en rima) Aqest pas es rimatz.

34. V. 34 (dunan nou : d'uno anno nuovo.

35. V. 36 (soferi) suffero.

36. V. 37 (destoli) destollo.

37. V. 40 (com qe laora) Quia hic quidem laborat et efforcet(?) la rima .laora. de gran dir.

38. V. 42 (Cel de mon clin odierna) Mainiers del castel de mondui(sic!) qamet tan fort Naudierna. na lamat plus dun uou en despeit de mi. tant am lis.

c. IoC zum Gedicht Sols sui qui soi (Can. XV).

39. V. 3 (selduis; sic) Selduis : subtraxerit.

40. V. 5 (epuois) $E$ puois : et postea.

41. V. 12 (cab uaus) ut dicit $G$. de borneill cab ... que per mans : non uado medio per ualles. et per plans et per puois : altitudines.

42. V. I3 trobaissi : ita trobem.

43. V. 18 (bels demors) aig(?) forset la rima. que degra esser ors estreida. demors bel demorar fa cum aquesta dompna. so es bel solazar e bel estar.

44. V. 26 und 27. (Que ges rozers peraiga etc.). Daurde dicit roeries . fluvius rodasi . rozers qui ingrossatur per aquas pluvias (?) et tum habet gran briu: forza. dotz so es la uena onde ue laiga e la fontana. on (P) se dilz. de uem de laiga de la dotz(?) so es daquella qades sortz.

45. V. 28 estanc quod dicimus stagnum. lacus propterea quod ibi aqua estancat se et ita stat. No faz estanc etc. et non facia estanc et simile(?) e no faza estanc. so uol dire Rodanus qui ingrossatur aquis non ita fortiter currit ubique spargitur quod ego plus lagrimis amorosis inondinationis (P) non faciam et maiorem lacum amoris. inde uoco (P) quod eam remiro.

46. V. 29 (bortz) Aqui 1 apellat bortz campis natus. so es de adulterio natus . quod dicimus ${ }^{2}$ Bastardo.

47..V. ? (giortz) : bagorda.

c. IOD Zum Gedicht Er uei uermeillz uerz (Can. XIII).

48. (V. 3) (Eil votz dels auzels sona e tint) dicit $G$. de borneill. $Q e$ fan retentir.

49. (gandirs) de gando, dis : fugio.

1 Der Glossator versucht vermutlich wie bei estanc eine etymologische Erklärung zu geben: 'borts ist ein dem $R$ ande [des Weges, vergl. unser „hinterm Gartenzaun“"] Geborener'.

2 Die Hs. giebt hier nur $q$. $d$., doch war ich nach dem kurz zuvor ausgeschriebenen quod dicimus zu dieser Auflösung berechtigt. 
50. V. 10 (sint) sentio.

5I. V. 13 (parcedors) per donadors. de parco, cis.

52. V. 14 (blandres) so es humilitatz.

53. (a enois) pro enois.

54. V. 17 (mint) : mentio.

55. V. 24 (uint) : $X X$.

56. V. 26 (va be sui fols) vadit bene: eu sui fols : uado quod din aqest treua et aital loc: briga.

57. V. 28 (tigris menandres) nomina fuuiorum.

58. V. 35 (poilla e flandres) nomina ciuitatum.

59. V. 36 (feingz juocs) ficticios iocos.

6o. V. 38 (cossint) consentit.

c. I I A zum Gedicht Doutz braitz ecritz (Can. XII).

6I. V. 8 (rimastrampa) desauinen.

62. V. 10 (ni nom presi destoutas) so es que nom destolgui dela dreita uia.

63. V. 19 (pecx) fatuus.

64. V. 23 (colobra) so es us serpens que pulet trop fort.

65. V. 24 (don tan mals motz escampa) de linguis quorum escampa : exit tan mals motz so es tans mal dires.

66. V. ? tampa a....

c. I B zum Gedicht En breu briscaral temps (Can. XI).

67. V. 17 (Faillirs esmendatz) Qui peca e menda etc.

68. V. 23 (Mais uolgra trar penels desertz etc.) magis uellem sustinere penam. ubi non ac daucel agre. so es nidum.

69. V. 43 (stug) custodio.

70. 1. 49/50 (Arnautz uol sos chanz sia offertz lai on dotz motz mouon agre) Aquesta sod estaua en un castel que es en la comtat de Peiregors. per so ditz que mouen : comenza en agre.

c. I I $\mathrm{C}$ zum Gedicht Autet e bas (Can. VIII).

71. V. 2 (renc) rengar o arengar. so es stare per ordinem. inde dicitur: Uns rencs de caualiers.

72. V. 17 (masauta) Abelis.

73. V. I 9 (Amors?) : ipsa mea domina.

74. V. 22 (sescanta) estinguat.

75. V. 23 (pels us) propter unos que fan graues uel grandes gemitus del altrui ioi.

76. V. 26 (en sa gauta?) cum amore.

77. V. 29 (en fadenc) so es en mateza.

78. V. 5I (et estrus) so es frens (?) et enbroncs..... di deo.

79. V. 53 (sauta) los cors.

8o. V. 52 (el cor teing prems) prems dicit quod pressum et calcatum cum pondere superiore. 
c. 2 A zum Gedicht Lo ferm uolers (Can. XVIII).

81. V. 2 (escoissendre) findere.

82. V. 5 (afrau) so es a furto a fraude.

83. V. 24 (com forts) quum homo fortis.

84. V. 35 (marma) quod nimiam habet cogitationem anima mea.

95. V. 40 (Son desirat) An Bertran de Born.ab cui se clamáua dessirat.

c. $4 \mathrm{I}$ C zum Gedicht Puois en Raimons (Can. I).

86. V. ? $O t$ : setet.

87. V. 34 fems so es leame. inde femoriens. locus ubi acceruatur fenis.

B. $\mathrm{Zu}$ anderen Dichtern $\mathbf{1}$ :

88. c. 3 B Sordello Dompna mieilz qon non pot. (ses cor vaire) senza fallacio.

89. c. $13 \mathrm{C}$ Peirols Nuillz hom non sauzi tan gen. (esmals non camenten) esi en als non men enten.

90. c. I4 A (Peirols Altressi col cignes fai, Qan uol morir chan. (Zu diesem Anfang) Ouidius libro epistularum:

Sic ubi fata uocant gelidis abiectus in erbis Ad uada menandri concinit albus color.

I. c. I 7 A Lo monges de poncibot Bens cuidet ueniar amors. (non es per que) meus... tortz.

92. c. I7 B Lo monges de poncibot ..... ( ) enneguna maneira.

93. c. $8 \mathrm{~A}$ Monges de poncibot Amors saus plagues. (quau ses mon pensamen) en aut le uos. quod est alsare.

94. c. $32 \mathrm{~A}$ Elias Cairels. (uoia?) fatua.

95. c. $34 \mathrm{~A}$ Elias Carels.

Garrics e Cassaignz idem est. Bens quem ...

Wir sind verpflichtet, wenigstens zu versuchen, den Autor dieser Glossen zu entdecken, die in ähnlicher Reichhaltigkeit sich wahrscheinlich in keiner anderen provenzalischen $\mathrm{Hs}$. finden werden. $\mathrm{Dafs}$ derselbe nicht ein Provenzale ist, wie man zunächst vermuten würde, sondern ganz entschieden nur ein Italiener sein kann, geht nicht blols aus dem Gebrauche einzelner italienischer Wörter und

1 Auf f. I I $^{\circ}$ befindet sich unten die Bemerkung:

Dreitz e raizon quieu chant em demori

Dritto e ragion chio canti et mi soggiorni.

von einer Hand des 16. Jahrh., vielleicht Barbieris. 
Wendungen, wie alegro, d'un anno nouo, cosa di uana gloria hervor, sondern noch mehr aus den Erklärungen von estanc und bortz durch quod dicimus stagnum und quod dicimus bastardo ${ }^{2}$, die augenscheinlich nur von einem Italiener herrühren können.

Ferner mufs dieser Italiener im Ausgang des 13. oder. in der ersten Hälfte des 14. Jahrh. gelebt haben, da der Schriftcharakter der jener Zeit ist und die durchweg richtige Interpretation schwieriger Stellen eine Periode voraussetzen, in welcher die Kenntnis des Provenzalischen in Italien noch lebendig war, was später nicht mehr der Fall ist.

Drittens mufs derselbe zu den Gebildesten der Nation gehört haben. Denn aufserdem dafs er das Provenzalische beherrscht, schreibt er ein korrektes Latein. Ovids Heroiden sind ihm so geläufig, dafs er sie aus dem Gedächtnis citiert. 1 Überhaupt ist die Art, Parallelstellen aus Guiraut de Borneil und Daude de Pradas anzuführen und ferner bei Peirol die Stelle aus Ovid anzugeben, welche er nachgeahmt hat, nicht die eines gewöhnlichen Geistes.

Viertens, und das ist es, was mich zuerst frappiert hat, der Glossator muss eine ganz spezielle Vorliebe für Arnaut Daniel gehabt haben. Denn seine Bemerkungen beziehen sich, wie ich durch die Teilung leicht übersehbar gemacht habe, fast ausschliefslich auf diesen Dichter. Bei den übrigen hat er gelegentlich etwas an den Rand geschrieben, Arnauts Gedichte hat er eingehend studiert und sich über jede einzelne Stelle klar zu werden gesucht. Dieser Umstand kann nicht etwa durch die gröfsere Schwierigkeit von Arnauts Gedichten erklärt werden, welche einen Kommentar notwendiger machten, als die übrigen. Denn es werden auch einfache Verbalformen (tolli, queri etc.) und ganz gewöhnliche Wörter (e puois, masanta, a frau etc.) glossiert, die sich bei allen Dichtern vorfinden. Dies beweist von Neuem, dafs der Schreiber kein Provenzale war, denn ein solcher hätte, wenn er für sich selbst schrieb, derartiger Erklärungen nicht bedurft; und wenn er etwa, welche Annahme schon an sich wenig wahrscheinlich ist, Andern das Lesen der Hs. hätte erleichtern wollen, so hätte er die ganze Hs. mit Bemerkungen versehen und nicht gerade die schwierigsten Gedichte ausgewählt, um an ihnen elementare Dinge zu erklären. Vielmehr werden wir notwendig auf einen Italiener gewiesen, der das Studium mit Arnaut Daniel begann und an ihm besonders Gefallen fand.

Keiner nun scheint mir diesen verschiedenen Bedingungen entsprechen zu können als Dante oder etwa noch Petrarca. Dafs die Bemerkungen von dem Letzteren nicht geschrieben sind, kann ich bei meiner Vertrautheit mit seinen Schriftzügen auf das Bestimmteste versichern; bleibt also nur Dante. Dafs diese Persönlichkeit die beiden ersten Forderungen erfüllt, ist evident. Dafs

1 Keine der erhaltenen Hss. bietet nämlich gelidis in erbis, sondern alle udis in erbis. Man sieht leicht, wie sich in der Erinnerung das gewöhnlichere Wort an die Stelle des ungewöhnlichen setzte.

2 Dante gebraucht diesen Ausdruck Purg. XIV 99. 
ihm das Provenzalische ebensowie das Lateinische geläufig war, brauche ich den Lesern dieser Zeitschrift nicht darzuthun. Ebenso wenig, dafs er Ovid kannte. Darauf dafs die citierte Epistel Ovids ihm besonders geläufig sein mufste, weil sie einer der Hauptfiguren .der Aeneis, der Dido, in den Mund gelegt ist, weise ich nur beiläufig hin. Eine Bemerkung wie die quod dicimus stagnum statt stagno ist echt dantisch, denn Dante nennt sich nicht Italiener, sondern Lateiner. ${ }^{1}$ Oder vielmehr Lateinisch und Italienisch (volgare) sind ihm nur verschiedene Arten derselben Sprache. Das Italienische dient für den mündlichen Verkehr und für $\mathrm{Ge}-$ dichte, die auch für Frauen und Kinder bestimmt sind, für wissenschaftliche, und besonders grammatische Dinge empfiehlt sich der Gebrauch des Lateinischen, das auch deswegen den Namen gramatica führt. ${ }^{2}$ Mit dieser Theorie stimmt überein, dafs die grammatischen und lexikalischen Glossen zum Teil lateinisch sind; nur wo das Italienische ein dem zu erklärenden ganz nahestehendes Wort bot, wurde dasselbe begreiflicherweise nicht unterdrückt. Die provenzalischen Bemerkungen brauchen nicht zu derselben Zeit geschrieben zu sein, wie die übrigen, und sind es wahrscheinlich nicht, da sie, die entweder sachlich wie (No. 28, 38 u. s. w.) oder metrisch (No. 33, 43) sind, oder die Bedeutung seltener Wörter durch üblichere umschreiben, eine grölsere Kenntnis der provenzalischen Sprache voraussetzen, als die lateinischen Glossen verraten. Diese hat also Dante vermutlich zu einer Zeit niedergeschrieben, als ihm das Provenzaliche durch persönlichen Verkehr mit Troubadours vertraut geworden war und vielleicht sind sie auch dem Wortlaut nach so niedergeschrieben, wie sie aus dem Munde derselben flossen.

Fünftens. Dantes Verehrung für Arnaut Daniel ist bekannt, aber sie ist auch etwas ganz Spezifisches. Der der Wissenschaft leider viel zu früh entrissene Canello hebt in seinem Buche über Arnaut Daniel 3 vortrefflich hervor, wie die schwer verständliche Ausdrucksweise dieses Dichters ihm selbst in der Provence nur wenige Anhänger verschaffte. Und noch viel weniger fand er deren in Italien, wo sein Verständnis noch grö/sere Schwierigkeiten machte. Daher wählen die in Italien entstandenen Abhandlungen über die Dichtkunst nur sehr selten Beispiele aus ihm, während sie von ganz unbedeutenden Dichtern Dutzende anführen und dafs Arnaut von italienischen Dichtern vor Dante nachgeahmt worden wäre, davon sind nur verschwindend geringe Spuren nachzuweisen. Alles Zeugnisse, dafs Arnaut in Italien bis auf Dante fast unbekannt war, wie es Canello ausdrücklich ausspricht: dovrasi conchiudere che

1 Tertia quae latinorum est, se duobus privilegus attestatur pracesse etc. ... puta Cinus pistoriensis, et amicus eius. De Vulg. Elo. I 10.

2 De Vulg. El. I I und passim.

3 U. A. Canello, La vita e le opere del trovatore Arnaldo Daniello etc. Halle, Max Niemeyer 188.3, p. 42-44. 
la farma grande del nostro trovatore comincia dal secolo $X I V$, con Dante, ed ¿̀ ther massima parte opera di lui. 1

Aker noch mehr. Dals Dante im Purgatorium ${ }^{2}$ Arnaut nicht nur eire Reihe von Versen widmet, sondern ihn, und ihn ausschlief slch in der ganzen Dichtung ${ }^{3}$, provenzalisch sprechen lälstbedarf jei einem Dichter wie Dante, der sich zu einer solchen Ausnahme nicht ohne gewichtigen Grund entschlossen haben kann, meines Erachtens einer besonderen Erklärung. Die Annahme, dafs er damt blols seine Hochschätzung dieses Troubadours habe anzeigem vollen, Ist nicht zulässig, da er kurz vorher schon ausdrücklich voon ihm gesagt hatte:

\section{Versi d'amore e prose di romanzi \\ Soverchid tutti etc.,}

es hätte also zu diesem Zwecke der Auszeichnung nicht bedurft. Nimmt man dazu, dafs Arnaụt in demselben Gesang wie Guinicelli auftritt, den Dante als seinen Lehrer in der Dichtkunst betrachtete und dafs dieser, der selbst in einem Gedichte 4 Arnaut nachgeahmt hat, ihn auf den provenzalischen Troubadour verweist, der in se in er Sprache besseres geleistet habe, als er selbst, so kann dies doch nur heifsen, dafs Dante durch die Gedichte Guinicellis zum Studium Arnaut Daniels veranlafst worden sei und also, dafs er das Studium des Provenzenzalischen mit Arnaut begonnen habe. Deswegen lärst er ihn provenzalisch sprechen, weil er das Provenzalische aus ihm und durch ihn gelernt hat: es ist dies eine Art und Weise, seinen Dank abzustatten, die auch uns modernen Menschen nicht unverständlich ist. Und die lateinischen Glossen der $\mathrm{Hs} . \mathrm{H}$, besonders die zu den ersten Gedichten, zeigen uns in der That einen solchen Anfänger.

Alle diese Indizien zusammengenommen, ergeben einen nicht geringen Grad von Wahrscheinlichkeit für Dantes Autorschaft. Das in vielen Fällen entscheidende Beweismittel, das paläographische, kann hier nicht zur Anwendung kommen, da wir meines Wissens keine authentische Zeile von Dantes Hand besitzen. Aber eine Bemerkung Leonardo Brunis vermag uns hierfür wenigstens einigen Ersatz zu bieten. In seiner kurzen Biographie Dantes schien ihm die Schrift des Dichters einer besonderen Erwähnung wert, und er sagt; fu ancora scrittore perfetto ed era la lettera sua magra e lunga $e$ molto corretta, secondo io ho veduto in alcune pistole di sua propria man scritte. Nun, wenn ich die Kursiv (von dieser spricht Leonardo offenbar) der Randglossen charakterisieren sollte, ich wülste es nicht anders zu thun, als mit den Worten: sehr fein, länglich und kalligraphisch.

1 1. c. p. 44 .

2 Purg. XXVI I15-I 48.

3 z. B. nicht Bertian de Born, vor dem er, wie das Vulg. El. zeigt, doch zur Zeit der Abfassung der Commödie auch eine grofse Achtung besafs.

4 Ich meine die Canzone Lo fin pregio avanzato, vergl. Gaspary, Litter. Gesch. S. 103. 
Ich wiederhole, dafs ich nicht den Anspruch erhebe, einen vollgültigen Beweis für dies Autograph Dantes erbracht zu haben, und dieser wird vielleicht niemals zu erbringen sein. Kaum wird es jedoch möglich sein einen andern Kandidaten zu nennen, bei dem alle die angeführten Vorbedingungen noch besser zuträfen, als bei Dante.

Aber selbst wenn diese Glossen nicht von Dante herrührten, so würden sie doch nicht ohne Wert sein. Es sind einige unter ihnen, die, wenn sie uns auch nicht geradezu Neues lehren, doch bisher Vermutetes bestätigen und uns deshalb sehr erwünscht sein müssen. So bestätigt I. die Vermutung Canellos: tr aill deve dire 'traccia' 'vestigio'1, 5. die Erklärung Chabaneaus, dafs a es daill abzuteilen und dafs dies eine sprichwörtliche Redensart sei, die bedeute „ziellos umherirren“.2 4. bezeugt, dafs die naheliegende Ableitung noirim von nutrimen auch hier zutreffend ist. Deswegen sind die künstlichen Auslegungen Canellos abzuiehnen. Der Dichter sagt, wie mir scheint, in der vierten Strophe: Nicht zum Vergnügen wende ich mich von Euch ab, Geliebte, die ich verehre; sondern aus Furcht vor den Verrätern, vor denen der Genufs zittert (den Störenfrieden des Genusses), gebe ich mir den Anschein, als ob ich von Euch nichts wissen wollte; denn (noch) nie hatten wir von ihrer Ernährung Vergnügen ${ }^{3}$ : verflucht sei, wer sie (noirim) ihnen bietet.4

Der Anfang von Sim fos Amors ist gemäls der Note $8 \mathrm{zu}$ übersetzen: Wenn die Geliebte gegen mich so freigebig sein wollte, wie ich gegen sie mit meinem Herzen, so würde ich nicht in Verlegenheit geraten (oder Schulden zu machen brauchen), um grofses Liebesglück zu genielsen. ${ }^{5}$ Canello hat diese Stelle, die er in der Übersetzung (S. I 36) mit per la grandezza del bene ch' io cerco non opporrei difficoltà wiedergiebt, ganz milsverstanden; imbarcarsi heifst noch heute im Italienischen, das den Bedeutungsübergang klar veranschaulicht, zunächst „sich einschiffen“, dann "sich in eine Angelegenheit einlassen, etwas unternehmen“ schliefslich „Verpflichtungen eingehen". Weniger künstlich ausgedrückt, wäre also der Sinn unserer Stelle: ich liebe Euch aufrichtig und wahr und Eure Gunst, hehre Frau, würde mich so unendlich erfreuen, dafs ich mein Glück nicht bei andern $\mathrm{zu}$ suchen brauche. ${ }^{6}$ IO. und II. bestätigen Erklärungen Canellos. Die Glosse I 8 . unterstützt Bartschs Auffassung von detz als decem. Vielleicht darf man die schwierige

1 1. c. p. 197 .

2 bei Canello p. 188.

3 d.h. sie bezahlen ihre Parasitenexistenz durch an uns geübten Verrat. Und deshalb: Malmes que lor o cuoilla! Es ist kein Grund vorhanden, von dieser Lesart, die ABCGHIKNN² darbieten, abzuweichen, wie Canello thut.

1 wörtlich : für sie sammelt (von colhir).

5 darüber, dals per gran joi wahrscheinlich die ursprüngliche Lesart war, siehe unten.

6 Im Text aber spricht der Trobador, wie häufig, diese Worte nicht direkt aus, sondern richtet sie in monologischer Form an sich selbst, während er die Geliebte erst in der fünften Strophe anredet. 
Stelle wie folgt übersetzen: Geliebte, gieb Acht, dafs ich wohl aufgenommen werde ${ }^{1 ;}$ denn, wenn $\mathrm{Du}$ mich schlecht empfängst (mich zurückweisest), so fürchte ich, wirst $\mathrm{Du}$ soviel Schlechtes von mir erfahren (wörtlich: dafs ich so gegen alle zehn Gebote gehandelt habe), dals es besser ist, dals Du Dich dávon scheidest (es unterlälst). Dafs der Zusatz detz zu pecs ein sehr überflüssiger und nur dem Reime zu Liebe geschehen ist, deutet auch die Glosse durch ihr ut ita dixerim an. Áber dafs Jemand, der einen Reim auf etz sucht, bei peccata zunächst an decem denkt, ist begreiflich. Dagegen ist V. 74 gemäls Note 25 decs als „Ziel, Endpunkt“, aufzufassen.2

Während bei den Versen $82-85$ desselben Gedichtes Canello liest Can non amei Ren tan ab meins d'ufaut Anz vos desir Plus que Dieus cil de Doma ${ }^{3}$ und übersetzt 'che mai ho amato alcuna donna con più di sincerità $e$ vi ho in cuore più che Dio quella(P) di Doma' hat Chabaneau 4 durch seine Worte: Je lirais dans Arnaut: Anz vos dezir plus que $D_{e u}{ }^{5}$ cil (ou cel?) di Doma die richtige Auffassung angedeutet: denn ich habe nie eine Frau mit weniger Hochmut ${ }^{6}$ geliebt, ja, ich sehne mich mehr nach ihr, als die von Doma nach Gott. Was der Dichter hier betonen will, ist das Demütige seiner Liebe und so spricht er denselben Gedanken noch einmal an andrer Stelle $^{i}$ (XIV 25-27) aus:
Non sai hom tan sia e Dieu frems
Ermita ni monge in clerc
Cum ieu vas cella de cui can.

Die glückliche Vermutung Chabaneaus Dona est une petite ville du Perigord ou il a pu exister du temps d'Arnaut Daniel un monastive ou seulement un ermitage wird durch die Note 28 vollauf bestätigt. No. 38 schliefslich giebt über eine bisher unerklärt gebliebene Stelle Aufklärung. Dafs in den Versen $\left(\mathrm{X}_{4} \mathrm{I}-42\right)$ Canc plus non amet un ou Cel de Monclin Audierna eine Anspielung auf die Personen eines damals bekannten Ritterromans gesucht werden müsse, hat schon G. Paris (1. c. p. 227) mit Recht angenommen, aber er wufste dieselbe nicht nachzuweisen. Vielleicht gelingt es ihm jetzt, da die Note uns nicht nur die vermutlich richtige Form

1 Dals sui ben vengutz denselben Sinn hat, wie im Französischen être le bienvenu, ergiebt sich aus dem Gegensatz desacuoills. Nur sind wider Erwarten diese Worte nicht von bada abhängig gemacht, sondern das Verlangte wird bereits als Faktum hingestellt, was die Aufforderung kategorisch macht.

2 V. 73-76: denn Ihr seid das Ziel meiner thörichten Wünsche, über die von andern soviel gespottet wird.

3 Bartsch, Prov. Chrestomathie 4. Aufl. 1880 col. 137 liest: ans vos dezir plus que deus cil d'Edoma, was ich nicht zu deuten weifs.

1. c. $22 \mathrm{I}$.

5 Diese Lesart wird durch fast sämtliche Hs. unterstützt, die dieu oder deu, aber nicht den Nominativ bieten.

6 ufaut = ufana (s. Gl. 27), das mit „Hochmut" oder auch mit „Eitelkeit", zu übersetzen ist.

7 Welche übrigens auch Canello nicht entgangen ist (l, c. 221). 
Mondui ', sondern auch den Namen des Besitzers des Schlosses (Mainiers) darbietet.

Eine ganz besondere Beachtung aber verdienen No. 8 und No. 15, beide zu dem Gedichte Sim fos Amors. ${ }^{2}$ Hier wird zum Verse 3 eine Lesart citiert, die sich in keiner der erhaltenen Hss. vorfindet. Es bieten:
I. ABDL Ja per gran ben
II. IKN2 Jamais per ioi,
III. MMc Ja de mon ioi, $\mathrm{U}$ iai,
IV. $\mathrm{H}$ Ja de mon ior, $\mathrm{C}$ Ja de mos jors, f Ja de uos jors etc.

Canello ist bei diesem Liede zu einer bestimmten Klassifikation der Hss. nicht gelangt. Die Autorität von ABDL scheint deren Lesart als die ursprüngliche zu empfehlen. Wenn aber im Original ben gestanden hätte, so wäre es absolut unerklärlich, was die übrigen zahlreichen Hs., unter denen sich mehrere gute befinden, zu Änderungen veranlafst haben könnte und besonders wie sie von ben auf $i o i$, iai, ior und iorns hätten gelangen können. War dagegen die ursprüngliche Lesart dieselbe wie die der Glosse ia per gran ioi, so hat die erste Klasse augenscheinlich die Bedeutung von joi (= Liebesgenufs) nicht verstanden, und deshalb durch das triviale ben ersetzt, und die übrigen haben an der Verbindung von gran mit joi Anstols genommen. ${ }^{3}$ Der Fehler von $\mathrm{AB}$ mufs sich schon in deren unmittelbaren Vorlage (a) befunden haben, aus welcher sie auch $\mathrm{D}$ und $\mathrm{L}$, die bei diesen Gedichten einen kontaminierten Text bieten, geschöpft haben mögen. Der Glossator mufs also die ursprüngliche Lesart mindestens aus $\mathrm{x}^{1}$ geschöpft haben, $d . h$. aus dem Archetypus der Peire-Alvernhe-Handschriften. ${ }^{4}$

Dasselbe Resultat ergiebt die Gl. I5; V. 47 lautet

\section{$\mathrm{I}$ in $\mathrm{AB}$ Malastres $\left.\begin{array}{rl}\text { L } & \text { Malsacres } \\ \text { CQPS } & \text { Malsastres }\end{array}\right\}$ es queus ten, desconoissens}

II. DHIKMN2 E (car) per uos es cazutz pretz e iouens.

Die Lesart der zweiten Klasse kann in $x^{1}$ noch nicht vorhanden gewesen sein. $\mathrm{AB}$ stellt den Text dieser Quelle wahrscheinlich richtig dar, bis auf das erste Wort, das, wie alle übrigen

1 Mondui kann zwar ebenso leicht aus Monclin entstenden- sein, das die meisten Schreiber gelesen haben, als umgekehrt, aber es scheint, dafs der Glossator besser unterrichtet ist, als sie. Beachtenswert ist, dafs $\cdot R$, die hier Monclar hat, in einem Liede Vidals naudierna statt na Vierna geschrieben hat (s. Bartsch, Peire Vidal, Berlin 1857 p. I13). Ihr Name mufs also nicht unbekannt gewesen sein.

2 Diese werden auch von Canello citiert, nur dals bei ihm auf S. 178 und 180 die Buchstaben der Colonnen $H$ und $L$ vertauscht sind.

3 Es scheint mir auch nicht ausgeschlossen, dafs die Kopisten ioi gleich iocus (statt gaudium) gedeutet haben.

'S. Gröber, Die Liedersammlungen der Troubadours p. 479. 
Hss. der ersten Klasse zeigen, malsastres gelautet haben mufs. Und genau dies bietet der Glossator:

Malsastres es quieus ten desconoissens.

Also hat er, dem wahrscheinlich $\mathrm{H}$ gehörte, später den Archetypus der Peire-Alvernheschen für ein Lied vergleichen können. Wie stimmt dies zu Dante? Von ihm ist dasselbe wahrscheinlich. Arnaut muls er schon um I 290 studiert haben, da er sie in $A l$ poco giorno und andern in die neunziger Jahre fallenden Gedichten ${ }^{1}$ nachahmt, aber nichts nötigt uns anzunehmen, dafs er eine Peire-Alvernhesammlung, deren Kenntnis das de volgari eloquio voraussetzt, vor der Verbannung kennen gelernt habe.

Aber dies unscheinbare Faktum erhält eine nicht geringe Bedeutung für Dantes Biographie, wenn man bestimmen kann, wo sich $x^{1}$ befand und wo Dante Gelegenheit hatte, mit provenzalischen Troubadours in persönlichen Verkehr zu treten. Ich glaube dies zu können. Ich glaube ferner zu wissen, nicht nur dafs die Hs. H, welche von Grützmacher in das Ende des 14. Jahrh. gesetzt wird, mindestes zum Teil in das I3. Jahrh. gehört, sondern auch wer diesen Teil geschrieben hat. Doch dies zu entwickeln, dazu fehlt es mir gegenwärtig an Zeit und auch an den nötigen Büchern. ${ }^{2}$ Ich gedenke die provenzalischen Hss. des Vaticans noch eingehend zu behandeln, vielleicht auch sie ganz abzudrucken. Aber ich habe die Mitteilung der Randglossen nicht zurückbehalten wollen, um es andern zu ermöglichen, mit Hülfe derselben sicheren Dante-Autographen auf die Spur zu kommen.

1 S. Carducci, Studí letterari, p. 2 I I und Gaspary, Geschichte der Italienischen Literatur p. 27I.

2 Nicht einmal den dritten Band des Dante-Jahrbuchs, welcher Bartschs einschlägigen Artikel enthält, konnte ich erhalten.

A. Pakscher. 